\title{
Evacuación de unidades médicas del Instituto Mexicano del Seguro Social durante el 2018
}

\author{
Ana Lilia Bautista-Reyes, Juan Carlos Sánchez-Echeverría, Felipe Cruz-Vega, \\ Hermes Manuel Cortes-Meza y Jorge Loría-Castellanos* \\ División de Proyectos Especiales en Salud, IMSS, Ciudad de México, México
}

\section{Resumen}

Objetivo: Describir las características de la evacuación de unidades médicas del Instituto Mexicano del Seguro Social (IMSS) del 1 de enero al 31 de diciembre del 2018. Metodología: Estudio observacional, transversal, descriptivo y retrospectivo en el que se analizaron los escenarios de riesgo en los que se activó el protocolo de evacuación en las unidades médicas del IMSS durante 2018. Las variables analizadas fueron: tipo de unidad médica, tipo de evacuación (parcial o total), tipo amenaza que activó el protocolo, las áreas críticas evacuadas, protocolos de evacuación de la unidad médica y eventos adversos durante la evacuación, entre otros. Se empleó estadística descriptiva. Resultados: En el 2018 se evacuaron 26 unidades de atención médica del IMSS, predominando las de $2 .^{\circ}$ nivel (57.69\%) sobre aquellas del $1 .{ }^{\text {er }}$ (34.6\%) y $3 .{ }^{\text {er }}$ nivel (7.7\%) de atención; la evacuación parcial fue la más frecuente (61.53\%). La causa principal de amenaza fue de origen hidrometeorológico, en el $53.8 \%$ de los casos. Un 30.6\% de los casos ameritó la evacuación de áreas críticas. En el 38.4\% se requirió apoyo intersectorial. Solo seis unidades (23\%) contaban con Comité Hospitalario para Emergencias y Desastres. Aunque se presentaron eventos adversos durante el procedimiento, ninguno fue fatal. Conclusiones: La evacuación de un hospital es un escenario real que requiere un protocolo actualizado, probado y socializado, así como personal capacitado, para evitar muertes prevenibles.

Palabras clave: Desastres. Evacuación. Unidades médicas.

\section{Evacuation of medical units of the Mexican Social Security Institute during 2018}

\section{Abstract}

Objective: To describe the characteristics of the evacuation of the Mexican Social Security Institute (IMSS) medical units from January $1^{\text {st }}$ to December 31, 2018. Methodology: We performed an observational, cross-sectional, and descriptive design to analyze the risk scenarios in which the evacuation protocol was activated at IMSS medical units during 2018. The variables analyzed were type of medical unit, type of evacuation (partial or total), type of event or threat by whereby the protocol was activated, the evacuation of critical areas, evacuation protocols of the medical unit, and adverse events during the evacuation, among others. Descriptive statistics were used. Results: In 2018, 26 medical care units were evacuated, predominating those of the $2^{\text {nd }}$ care level $(57.6 \%)$ over those of the $1^{\text {st }}(34.6 \%)$ and $3^{\text {rd }}(7.7 \%)$ levels of care; partial evacuation

\section{Correspondencia}

*Jorge Loría-Castellanos

E-mail: jloriac@ @otmail.com
Disponible en internet: 06-11-2019 Rev Educ Investig Emer. 2019;1(3):82-88 www.medicinadeemergencias.com (1) Eedicina de Emergencias, AC. Publicado por Permanyer México SA de CV. Este es un artículo open access bajo la licencia CC BY-NC-ND (http://creativecommons.org/licenses/by-nc-nd/4.0/). 
was more frequent (61.5\%). The main threat was of hydrometeorological origin, with $53.8 \%$ of cases. The $30.6 \%$ of cases required the evacuation of critical areas. In $38.46 \%$, intersectoral support was required. Only six medical units (23\%) had a Hospital Committee for Emergencies and Disasters. Although there were adverse events during the procedure, none was fatal. Conclusions: The evacuation of a hospital is a real scenario that requires an updated, tested and socialized protocol to avoid preventable deaths, as well as trained personnel.

Key words: Disasters. Evacuation. Medical units.

\section{Antecedentes}

El fortalecimiento de los preparativos en los hospitales para responder ante cualquier amenaza o evento debe considerar todos los escenarios de riesgo posibles, así como establecer protocolos y procedimientos que permitan ejecutar una respuesta eficiente y oportuna para evitar pérdidas humanas ${ }^{1}$. América fue la segunda región más afectada por desastres entre el 2004 y 2013, con alrededor de 98 millones de víctimas. El 77\% de los establecimientos de salud en la región se encuentran ubicados en áreas susceptibles al impacto de un evento $o$ amenaza que pongan en riesgo su funcionamiento. Por cada establecimiento de salud que deja de funcionar en un escenario de emergencia o desastre, alrededor de 200,000 personas quedan sin acceso a los servicios de salud, justo cuando más lo requieren².

En este contexto, el Instituto Mexicano del Seguro Social (IMSS), con base en su experiencia en emergencias y desastres, desarrolló un protocolo para gestionar de forma anticipada las necesidades para evacuar un hospital y con ello garantizar la continuidad de sus operaciones, y salvaguardar la vida de los pacientes y de su personal. Este protocolo debe ser probado y socializado, e incluir los procedimientos necesarios para que todas las áreas del hospital puedan ser evacuadas de forma rápida y segura. Por ello se propuso realizar los ajustes pertinentes a los planes de respuesta de los hospitales, con un enfoque multiamenaza (que considere todos los escenarios de riesgo), centrado en las personas, inclusivo, y que permita tomar la decisión de evacuar la edificación con base en una rápida evaluación de riesgos ${ }^{3-5}$.

El riesgo durante una evacuación es mayor cuando se trata de áreas o espacios con pacientes que debido a la gravedad en su estado de salud tienen mayores requerimientos de personal, equipo biomédico especializado 0 algún procedimiento terapéutico. Estas áreas del hospital, por sus características y nivel de complejidad de atención, se definen como "áreas críticas» ${ }^{3,4}$. La evacuación de estas áreas representa un verdadero reto; el no contar con una preparación y capacitación adecuada puede disparar una crisis en la organización e incrementar el riesgo de lesiones y muertes potenciales evitables ${ }^{6}$.

Un elemento para fortalecer la capacidad de respuesta de un hospital es establecer acuerdos y convenios de colaboración interinstitucionales e intersectoriales, para contar con una red de apoyo logístico y de operaciones en salud que garantice la seguridad de los pacientes $^{1,2}$. La necesidad de evacuar establecimientos de salud se debe considerar en la Gestión Integral del Riesgo de Desastres en Hospitales, incluida en el análisis estratégico de riesgos, mediante la implementación de protocolos dentro de los planes hospitalarios ${ }^{7}$.

El objetivo central de este trabajo es describir las principales características de la evacuación de unidades hospitalarias del IMSS (el mayor proveedor de atención médica en México) durante el 2018.

\section{Metodología}

Se realizó un estudio observacional, transversal, descriptivo, y retrospectivo en el que se analizaron los casos de evacuación de unidades médicas del IMSS del 1 de enero al 31 de diciembre del 2018. Las variables evaluadas fueron: tipo de unidad médica evacuada (1. $.^{\text {er }}, 2 .^{\circ}$ o $3^{\text {er }}$ nivel de atención), tipo de evacuación (parcial o total), momento de la evacuación (mes, día, turno), tipo de amenaza, evacuación de áreas críticas (urgencias, quirófano, tococirugía, terapia intensiva neonatal, terapia intensiva adultos, hemodiálisis), tipo de apoyo para la evacuación (por unidades médicas IMSS, no IMSS o intersectorial), personal que emitió la indicación de evacuación, protocolos de evacuación de la unidad médica, capacitación del personal en protocolos de evacuación y defunciones derivadas de la evacuación.

Los datos se obtuvieron del registro y seguimiento de las notificaciones en tiempo real que realiza el Centro Virtual de Operaciones en Emergencias y Desastres (CVOED, http://cvoed.imss.gob.mx) del IMSS, por personal médico capacitado en atender, coordinar y apoyar $^{3}$ a los responsables de ejecutar la respuesta en los hospitales ante una emergencia o desastre, derivada de 


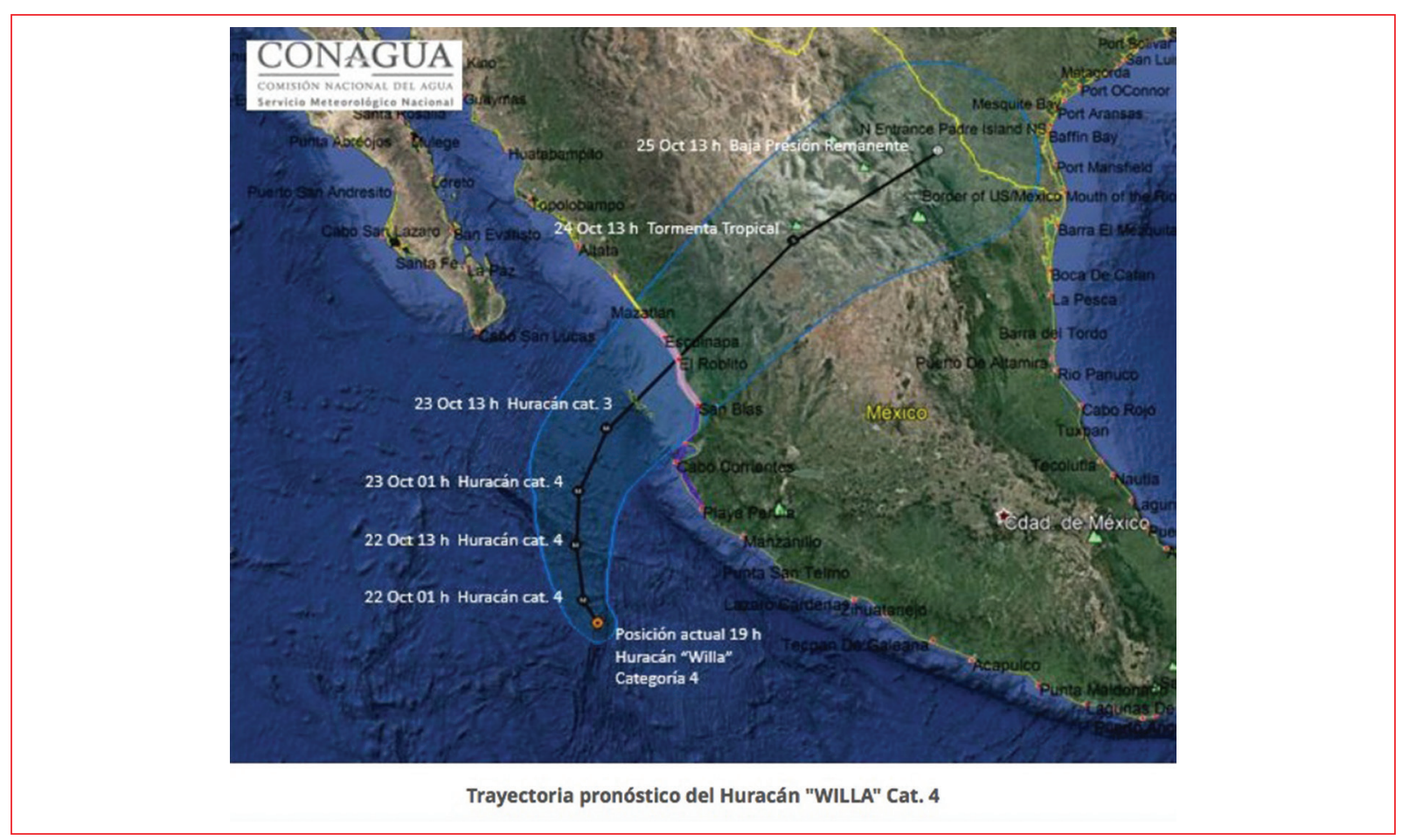

Figura 1. Mapa que muestra la trayectoria de un huracán. Ejemplo de amenaza de origen hidrometeorológico. Aviso 14, emisión 19:15 h, 21 de octubre del 2018 (Fuente: https://smn.cna.gob.mx/es/?option = com_visforms\&view = visformsdata\&layout $=$ data\&id $=107 \&$ cid $=2735$ ).

cualquier tipo de amenaza o evento, independientemente de su origen o magnitud. EI CVOED es un sistema informático que permite la comunicación directa en tiempo real desde las zonas afectadas por una crisis, emergencia o desastre, hasta los niveles directivos formando una línea de comunicación y coordinación. Integra los mapas de georreferencia de todas las unidades de atención médica a lo largo del país y permite emitir una alerta temprana a los hospitales que pueden ser afectados por una amenaza, así como herramientas útiles durante todas las fases de una emergencia o desastre, tales como censo nominal de pacientes, camas y servicios, hemoderivados, y directorios intrainstitucionales y extrainstitucionales, entre otros ${ }^{3-5}$. En el caso de las amenazas de origen natural, en las delegaciones afectadas por ciclones tropicales, el monitoreo y enlace comenzó desde los primeros avisos emitidos por el Servicio Meteorológico Nacional (Fig. 1), se trazaron mapas de amenazas tomando como base las proyecciones realizadas por los meteorólogos, utilizando una herramienta de georreferencia inserta en el sistema CVOED, y se reforzaron las líneas de comunicación en ambos sentidos mediante esta herramienta informática y por medio de redes sociales (Fig. 2).
Los datos obtenidos se analizaron empleando estadística descriptiva, las variables categóricas se representaron en frecuencias $(n)$ y porcentajes (\%).

\section{Resultados}

Del 1 de enero al 31 de diciembre del 2018 se evacuaron 26 unidades de atención médica del IMSS, el $34.6 \%(n=9)$ fueron unidades de primer nivel, el $57.7 \%$ $(n=15)$ de segundo nivel y el $7.7 \%(n=2)$ de tercer nivel (Tabla 1). Las unidades de atención médica que fueron evacuadas correspondieron a $15(41.7 \%)$ de las 36 delegaciones que tiene el IMSS en todo el país. Los eventos se presentaron con mayor frecuencia en los meses de octubre (46.2\%) y septiembre (19.2\%), los días lunes (53.8\%) seguido del jueves (15.4\%), y durante los turnos matutino $(65.4 \%)$ y nocturno $(23.1 \%)$. En el $61.5 \%$ de los casos $(n=16)$ las evacuaciones fueron parciales. El $30.6 \%$ de los casos $(n=8)$ incluyó la evacuación de áreas críticas: quirófanos $(n=5)$, urgencias $(n=4)$, tococirugía $(n=3)$, terapia intensiva neonatal $(n=2)$, terapia intensiva adultos $(n=2)$, y hemodiálisis $(n=2)$. El 53.8\% $(n=14)$ de las evacuaciones se produjo por amenazas naturales, subtipo 


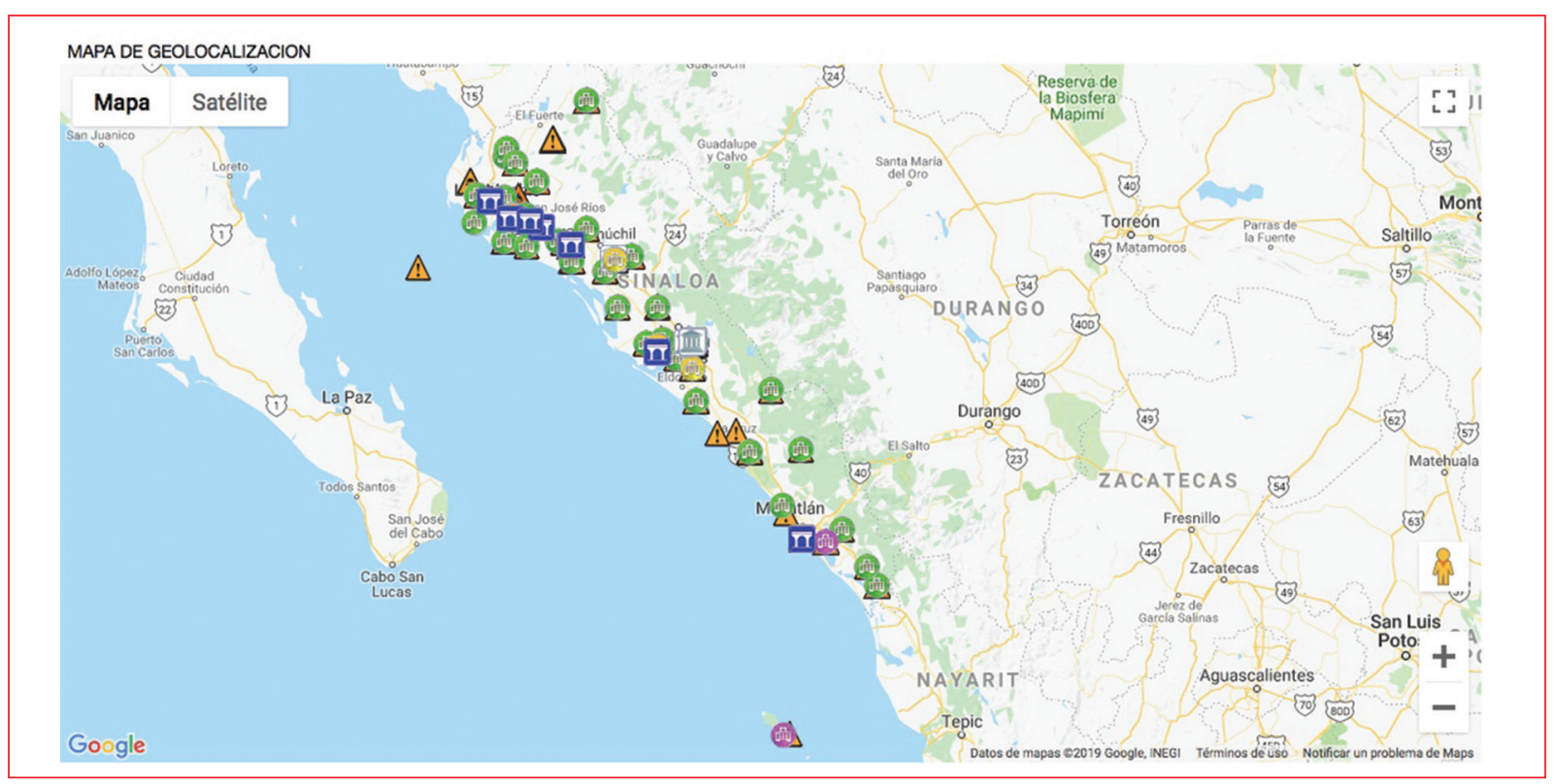

Figura 2. Mapa del Centro Virtual de Operaciones en Emergencias y Desastres. Ejemplo de una herramienta para alerta temprana, mediante la georreferencia de posibles unidades afectadas por el Huracán Willa, categoría 1 (Fuente: http://cvoed.imss.gob.mx/COED/home/standard/mapas/nacional/mapa_unidades_operativas.php).

hidrometeorológicas, nueve fueron totales y cinco parciales, dos de ellas incluyeron áreas críticas. Las amenazas restantes $(46.2 \%, n=12)$ fueron generadas por el ser humano, subtipo tecnológicas, nueve correspondieron a incendios y tres a materiales peligrosos, se requirió la evacuación parcial en dos ocasiones, que involucraron áreas críticas.

En el $15.3 \%$ de los casos $(n=4)$ se requirió el apoyo de unidades médicas del IMSS, en otro 15.3\% $(n=4)$ el apoyo fue por unidades no IMSS (Hospital privado, Secretaría de Salud Estatal, Hospital Naval, Instituto de Seguridad y Servicios Sociales de los Trabajadores del Estado), y en el $38.4 \%(n=10)$ se requirió apoyo intersectorial (Protección Civil, Bomberos, Policía Municipal, Cruz Roja, Cruz Ámbar). Protección Civil prestó con mayor frecuencia apoyo en 15 ocasiones, seguido de Bomberos en ocho eventos, Policía Municipal y Cruz Roja en dos ocasiones cada una. En 11 de los casos se combinó el apoyo de las dependencias.

Las evacuaciones fueron indicadas con mayor frecuencia por el subdirector en turno en el $26.9 \%(n=7)$, el director de la unidad en el $23.1 \%(n=6)$, el jefe delegacional de servicios de prestaciones médicas en el $23.1 \%(n=6)$, en el $7.7 \%(n=2)$ por el personal de los servicios y en el $3.8 \%(n=1)$ por el coordinador de brigada de evacuación. Solo el 23.1\% $(n=6)$ de las unidades médicas contaban con un Comité Hospitalario para Emergencias y Desastres, Plan Hospitalario para emergencias y Desastres, y Programa Interno de Protección Civil. El 46.2\% $(n=12)$ de las unidades evacuadas refirieron contar con personal capacitado en el tema de emergencias y desastres. Seis $(75 \%)$ de las ocho unidades que evacuaron áreas críticas contaban con personal capacitado en temas de emergencias y desastres o por el programa Hospital Seguro. No hubo defunciones derivadas de las evacuaciones, aun cuando en algunos casos no se implementó el protocolo.

\section{Discusión}

Este estudio se constituye como un primer acercamiento al registro de evacuaciones de unidades hospitalarias en México.

Aunque la gran mayoría de los textos refieren que no es deseable la evacuación de unidades hospitalarias, no obstante, como se demuestra en el presente documento, es una necesidad real ante múltiples escenarios de riesgo $0^{1,2,8}$.

Como se puede observar, estos eventos involucran a casi la mitad de los estados del país y a los tres niveles de atención, lo que puede afectar a pacientes con una diversidad de patologías y estados de gravedad, generando un fenómeno que pudiéramos considerar «universal» y en el que cada proceso de evacuación debe desarrollarse con las características 
Rev Educ Investig Emer. 2019;1(3)

Tabla 1. Características de las unidades médicas evacuadas del IMSS en 2018

\begin{tabular}{|c|c|c|c|c|c|c|c|}
\hline $\begin{array}{l}\text { Unidad } \\
\text { Médica }\end{array}$ & $\begin{array}{l}\text { Delegación } \\
\text { IMSS }\end{array}$ & $\begin{array}{l}\text { Nivel de } \\
\text { atención }\end{array}$ & $\begin{array}{l}\text { Tipo de } \\
\text { evento }\end{array}$ & Subtipo & $\begin{array}{l}\text { Evacuación } \\
\text { total o } \\
\text { parcial }\end{array}$ & $\begin{array}{c}\text { Número de } \\
\text { áreas } \\
\text { críticas } \\
\text { evacuadas }\end{array}$ & $\begin{array}{l}\text { Áreas críticas } \\
\text { evacuadas }\end{array}$ \\
\hline $\begin{array}{l}\text { Hospital } \\
\text { General de } \\
\text { Zona }\end{array}$ & Veracruz Sur & $2 .^{\circ}$ & $\begin{array}{l}\text { Amenazas } \\
\text { causadas } \\
\text { por el ser } \\
\text { humano }\end{array}$ & $\begin{array}{l}\text { Amenaza } \\
\text { tecnológica }\end{array}$ & Parcial & 3 & $\begin{array}{l}\text { Urgencias, } \\
\text { quirófano, } \\
\text { hemodiálisis }\end{array}$ \\
\hline $\begin{array}{l}\text { Unidad } \\
\text { Médica de } \\
\text { Alta } \\
\text { Especialidad }\end{array}$ & $\begin{array}{l}\text { Ciudad de } \\
\text { México Sur }\end{array}$ & $3 .^{\circ}$ & $\begin{array}{l}\text { Amenazas } \\
\text { naturales }\end{array}$ & $\begin{array}{l}\text { Amenazas } \\
\text { hidrometeorológicas }\end{array}$ & Total & NA & NA \\
\hline $\begin{array}{l}\text { Hospital } \\
\text { General } \\
\text { Regional }\end{array}$ & $\begin{array}{l}\text { Estado de } \\
\text { México Oriente }\end{array}$ & $2 .^{\circ}$ & $\begin{array}{l}\text { Amenazas } \\
\text { causadas } \\
\text { por el ser } \\
\text { humano }\end{array}$ & $\begin{array}{l}\text { Amenaza } \\
\text { tecnológica }\end{array}$ & Parcial & 1 & UCIN \\
\hline $\begin{array}{l}\text { Hospital } \\
\text { General } \\
\text { Regional }\end{array}$ & Baja California & $2 .^{\circ}$ & $\begin{array}{l}\text { Amenazas } \\
\text { causadas } \\
\text { por el ser } \\
\text { humano }\end{array}$ & $\begin{array}{l}\text { Amenaza } \\
\text { tecnológica }\end{array}$ & Parcial & 5 & $\begin{array}{l}\text { UCIA, UCIN, } \\
\text { quirófano, } \\
\text { urgencias, } \\
\text { tococirugía }\end{array}$ \\
\hline $\begin{array}{l}\text { Hospital } \\
\text { General de } \\
\text { Zona }\end{array}$ & Sonora & $2 .^{\circ}$ & $\begin{array}{l}\text { Amenazas } \\
\text { causadas } \\
\text { por el ser } \\
\text { humano }\end{array}$ & $\begin{array}{l}\text { Amenaza } \\
\text { tecnológica }\end{array}$ & Parcial & 2 & $\begin{array}{l}\text { Tococirugía, } \\
\text { quirófano }\end{array}$ \\
\hline $\begin{array}{l}\text { Hospital } \\
\text { General de } \\
\text { Zona }\end{array}$ & Veracruz Norte & $2 .^{\circ}$ & $\begin{array}{l}\text { Amenazas } \\
\text { causadas } \\
\text { por el ser } \\
\text { humano }\end{array}$ & $\begin{array}{l}\text { Amenaza } \\
\text { tecnológica }\end{array}$ & Parcial & 3 & $\begin{array}{l}\text { Quirófano, } \\
\text { sala de } \\
\text { recuperación, } \\
\text { tococirugía }\end{array}$ \\
\hline $\begin{array}{l}\text { Hospital } \\
\text { General de } \\
\text { Zona }\end{array}$ & Veracruz Sur & $2 .^{\circ}$ & $\begin{array}{l}\text { Amenazas } \\
\text { causadas } \\
\text { por el ser } \\
\text { humano }\end{array}$ & $\begin{array}{l}\text { Amenaza } \\
\text { tecnológica }\end{array}$ & Parcial & 1 & Quirófanos \\
\hline $\begin{array}{l}\text { Unidad de } \\
\text { Medicina } \\
\text { Familiar }\end{array}$ & $\begin{array}{l}\text { Baja California } \\
\text { Sur }\end{array}$ & $1 .^{\circ}$ & $\begin{array}{l}\text { Amenazas } \\
\text { naturales }\end{array}$ & $\begin{array}{l}\text { Amenazas } \\
\text { hidrometeorológicas }\end{array}$ & Parcial & NA & NA \\
\hline $\begin{array}{l}\text { Unidad de } \\
\text { Medicina } \\
\text { Familiar }\end{array}$ & Sinaloa & $1 .^{\circ}$ & $\begin{array}{l}\text { Amenazas } \\
\text { naturales }\end{array}$ & $\begin{array}{l}\text { Amenazas } \\
\text { hidrometeorológicas }\end{array}$ & Total & NA & NA \\
\hline $\begin{array}{l}\text { Unidad } \\
\text { Médica Rural }\end{array}$ & Sinaloa & $1 .^{\circ}$ & $\begin{array}{l}\text { Amenazas } \\
\text { naturales }\end{array}$ & $\begin{array}{l}\text { Amenazas } \\
\text { hidrometeorológicas }\end{array}$ & Total & NA & NA \\
\hline $\begin{array}{l}\text { Hospital } \\
\text { General de } \\
\text { Zona }\end{array}$ & Aguascalientes & $2 .^{\circ}$ & $\begin{array}{l}\text { Amenazas } \\
\text { causadas } \\
\text { por el ser } \\
\text { humano }\end{array}$ & $\begin{array}{l}\text { Amenaza } \\
\text { tecnológica }\end{array}$ & Parcial & 0 & No \\
\hline $\begin{array}{l}\text { Unidad de } \\
\text { Medicina } \\
\text { Familiar }\end{array}$ & Baja California & $1 .^{\circ}$ & $\begin{array}{l}\text { Amenazas } \\
\text { naturales }\end{array}$ & $\begin{array}{l}\text { Amenazas } \\
\text { hidrometeorológicas }\end{array}$ & Parcial & NA & NA \\
\hline $\begin{array}{l}\text { Unidad de } \\
\text { Medicina } \\
\text { Familiar }\end{array}$ & $\begin{array}{l}\text { Estado de } \\
\text { México } \\
\text { Poniente }\end{array}$ & $1 .^{\circ}$ & $\begin{array}{l}\text { Amenazas } \\
\text { causadas } \\
\text { por el ser } \\
\text { humano }\end{array}$ & $\begin{array}{l}\text { Amenaza } \\
\text { tecnológica }\end{array}$ & Total & NA & NA \\
\hline $\begin{array}{l}\text { Hospital } \\
\text { General de } \\
\text { Zona }\end{array}$ & Campeche & $2 .^{\circ}$ & $\begin{array}{l}\text { Amenazas } \\
\text { causadas } \\
\text { por el ser } \\
\text { humano }\end{array}$ & $\begin{array}{l}\text { Amenaza } \\
\text { tecnológica }\end{array}$ & Parcial & 0 & No \\
\hline
\end{tabular}


Tabla 1. Características de las unidades médicas evacuadas del IMSS en 2018 (Continuación)

\begin{tabular}{|c|c|c|c|c|c|c|c|}
\hline $\begin{array}{l}\text { Unidad } \\
\text { Médica }\end{array}$ & $\begin{array}{l}\text { Delegación } \\
\text { IMSS }\end{array}$ & $\begin{array}{l}\text { Nivel de } \\
\text { atención }\end{array}$ & $\begin{array}{l}\text { Tipo de } \\
\text { evento }\end{array}$ & Subtipo & $\begin{array}{l}\text { Evacuación } \\
\text { total } 0 \\
\text { parcial }\end{array}$ & $\begin{array}{c}\text { Número de } \\
\text { áreas } \\
\text { críticas } \\
\text { evacuadas }\end{array}$ & $\begin{array}{l}\text { Áreas críticas } \\
\text { evacuadas }\end{array}$ \\
\hline $\begin{array}{l}\text { Hospital } \\
\text { General de } \\
\text { Zona }\end{array}$ & Coahuila & $2 .^{\circ}$ & $\begin{array}{l}\text { Amenazas } \\
\text { naturales }\end{array}$ & $\begin{array}{l}\text { Amenazas } \\
\text { hidrometeorológicas }\end{array}$ & Parcial & 1 & Urgencias \\
\hline $\begin{array}{l}\text { Hospital } \\
\text { General de } \\
\text { Subzona }\end{array}$ & Coahuila & $2 .^{\circ}$ & $\begin{array}{l}\text { Amenazas } \\
\text { naturales }\end{array}$ & $\begin{array}{l}\text { Amenazas } \\
\text { hidrometeorológicas }\end{array}$ & Parcial & 0 & 0 \\
\hline $\begin{array}{l}\text { Hospital } \\
\text { General } \\
\text { Regional }\end{array}$ & Chihuahua & $2 .^{\circ}$ & $\begin{array}{l}\text { Amenazas } \\
\text { causadas } \\
\text { por el ser } \\
\text { humano }\end{array}$ & $\begin{array}{l}\text { Amenaza } \\
\text { tecnológica }\end{array}$ & Parcial & 0 & No \\
\hline $\begin{array}{l}\text { Hospital } \\
\text { General de } \\
\text { Subzona }\end{array}$ & Nayarit & $2 .^{\circ}$ & $\begin{array}{l}\text { Amenazas } \\
\text { naturales }\end{array}$ & $\begin{array}{l}\text { Amenazas } \\
\text { hidrometeorológicas }\end{array}$ & Total & NA & NA \\
\hline $\begin{array}{l}\text { Unidad de } \\
\text { Medicina } \\
\text { Familiar }\end{array}$ & Nayarit & $1 .^{\circ}$ & $\begin{array}{l}\text { Amenazas } \\
\text { naturales }\end{array}$ & $\begin{array}{l}\text { Amenazas } \\
\text { hidrometeorológicas }\end{array}$ & Total & NA & NA \\
\hline $\begin{array}{l}\text { Unidad de } \\
\text { Medicina } \\
\text { Familiar }\end{array}$ & Nayarit & $1 .^{\circ}$ & $\begin{array}{l}\text { Amenazas } \\
\text { naturales }\end{array}$ & $\begin{array}{l}\text { Amenazas } \\
\text { hidrometeorológicas }\end{array}$ & Total & NA & NA \\
\hline $\begin{array}{l}\text { Hospital } \\
\text { Rural }\end{array}$ & Sinaloa & $2 .^{\circ}$ & $\begin{array}{l}\text { Amenazas } \\
\text { naturales }\end{array}$ & $\begin{array}{l}\text { Amenazas } \\
\text { hidrometeorológicas }\end{array}$ & Total & NA & NA \\
\hline $\begin{array}{l}\text { Unidad } \\
\text { Médica Rural }\end{array}$ & Sinaloa & $1 .^{\circ}$ & $\begin{array}{l}\text { Amenazas } \\
\text { naturales }\end{array}$ & $\begin{array}{l}\text { Amenazas } \\
\text { hidrometeorológicas }\end{array}$ & Total & NA & NA \\
\hline $\begin{array}{l}\text { Unidad } \\
\text { Médica Rural }\end{array}$ & $\begin{array}{l}\text { Ciudad de } \\
\text { México Sur }\end{array}$ & $1 .^{\circ}$ & $\begin{array}{l}\text { Amenazas } \\
\text { naturales }\end{array}$ & $\begin{array}{l}\text { Amenazas } \\
\text { hidrometeorológicas }\end{array}$ & Total & NA & NA \\
\hline $\begin{array}{l}\text { Unidad } \\
\text { Médica de } \\
\text { Alta } \\
\text { Especialidad }\end{array}$ & $\begin{array}{l}\text { Ciudad de } \\
\text { México Sur }\end{array}$ & $3 .^{\circ}$ & $\begin{array}{l}\text { Amenazas } \\
\text { causadas } \\
\text { por el ser } \\
\text { humano }\end{array}$ & $\begin{array}{l}\text { Amenaza } \\
\text { tecnológica }\end{array}$ & Parcial & 0 & No \\
\hline $\begin{array}{l}\text { Hospital } \\
\text { General de } \\
\text { Zona }\end{array}$ & Tabasco & $2 .^{\circ}$ & $\begin{array}{l}\text { Amenazas } \\
\text { naturales }\end{array}$ & $\begin{array}{l}\text { Amenazas } \\
\text { hidrometeorológicas }\end{array}$ & Parcial & 4 & $\begin{array}{l}\text { Urgencias, } \\
\text { tococirugía, } \\
\text { quirófano, } \\
\text { hemodiálisis }\end{array}$ \\
\hline $\begin{array}{l}\text { Hospital } \\
\text { General de } \\
\text { Zona }\end{array}$ & $\begin{array}{l}\text { Estado de } \\
\text { México Oriente }\end{array}$ & $2 .^{\circ}$ & $\begin{array}{l}\text { Amenazas } \\
\text { causadas } \\
\text { por el ser } \\
\text { humano }\end{array}$ & $\begin{array}{l}\text { Amenaza } \\
\text { tecnológica }\end{array}$ & Parcial & 0 & No \\
\hline
\end{tabular}

UCIA: Unidad de Cuidados Intensivos de Adultos; UCIN: Unidad de Cuidados Intensivos Neonatales.

especiales de estos pacientes, así como de las propias unidades s, $^{1,8,9}$.

Si bien es cierto que la mayoría de las evacuaciones fueron parciales, se necesita contar con personal capacitado y planes específicos, a fin de optimizar los procesos y limitar las complicaciones, para aquellos escenarios en los que se requiera evacuar por completo la unidad, ya que esto demandará una mayor cantidad de recursos 3,10 .
No es de extrañar que las amenazas naturales ocasionaran la mayoría de los eventos, esto es debido a los riesgos hidrometeorológicos a los que está expuesto nuestro país. Sin embargo, resulta una preocupación la elevada frecuencia de eventos derivados por amenazas humanas, situación en la que en definitiva se ven inmersas las características tecnológicas y de violencia prevalentes, y que en la actualidad generan nuevas amenazas que gestionar ${ }^{11,12}$. 
En nuestro estudio, un punto a recalcar fue la necesidad de evacuar áreas críticas, proceso que per se requiere que el personal multidisciplinario (médico, enfermería, técnico, administrativo, etc.), no solo esté capacitado y se involucre de los procesos, sino que dichos procesos estén bien desarrollados, socializados y puestos a prueba con simulaciones y simulacros previos, y que estos planes consideren las características de infraestructura y personal propios de cada unidad $^{3,4}$.

Es una realidad que durante una evacuación las unidades se encuentran sobrepasadas, por lo que es crucial que existan dentro de los planes hospitalarios lineamientos de colaboración intrainstitucionales y extrainstitucionales que disminuyan los tiempos de reacción y optimicen la movilización de pacientes ${ }^{13,14}$.

Aunque las evacuaciones fueron mayormente indicadas por las máximas autoridades en el momento (jefes de delegacionales de prestaciones médicas, director o subdirector), llama la atención que en cuatro ocasiones fueron indicadas por personal sin esta autoridad (coordinador de brigadas, jefe de servicio e incluso el propio personal), situación que pudiera evidenciar la falta de implementación del protocolo, pudiendo derivar en complicaciones que comprometen la integridad de los pacientes y trabajadores ${ }^{10,14}$.

En más de la tercera parte de los casos se contó con apoyo intersectorial, principalmente de Protección Civil (además de Bomberos y Cruz Roja). Se debe considerar si este se dio "de buena fe» o porque se tenían establecidos acuerdos de cooperación o colaboración dentro de los programas específicos de respuesta ${ }^{10,14}$.

Para la adecuada gestión de riesgos y respuesta ante una emergencia $y / 0$ desastre que derive en una evacuación, se requiere contar con un Comité Hospitalario para Emergencias y Desastres que conozca los protocolos insertos en su plan de respuesta, y un Plan Hospitalario para Emergencias y Desastres, además del Programa Interno de Protección Civil implementado y actualizado ${ }^{10,14}$. Solo el $33.33 \%$ de las unidades de atención médica contaban con personal capacitado en temas de emergencias y desastres, y solo el 23\% tenía un Plan Hospitalario para Emergencias y Desastres, hechos que en definitiva influyen negativamente en el proceso de evacuación y en la seguridad e integridad de los pacientes y del personal ${ }^{14}$.

Un área de oportunidad será el analizar en un estudio posterior si existe relación entre el tipo de evacuación (parcial vs. total) y los turnos, quién indicó la evacuación y el contar o no con personal capacitado y planes hospitalarios, así como si la respuesta realizada se correlaciona con la forma de preparación $n^{10,14}$.

En ello nos encontramos trabajando.

\section{Conclusiones}

- Es una realidad la presencia de evacuaciones en unidades médicas y por ello la necesidad de contar con protocolos actualizados, probados y socializados que fortalezcan la capacidad de preparación para la respuesta.

- Se debe tener personal capacitado y planes acordes para poder realizar estas evacuaciones sin complicaciones o defunciones de los participantes.

- Es indispensable contar con un análisis estratégico de riesgos en cada hospital, dentro de su programa de gestión integral de riesgos, donde invariablemente debe incluirse la participación interinstitucional e intersectorial en la búsqueda de disminuir al máximo la vulnerabilidad de las unidades de atención médica.

\section{Bibliografía}

1. Marco de Sendai para la Reducción del Riesgo de desastres 2015-2030 [Internet]. Ginebra: Organización de las Naciones Unidas; 2015.

2. Organización Panamericana de la Salud, Organización Mundial de la Salud, 68. ${ }^{a}$ Sesión del Comité Regional de la OMS para las américas. Washington D.C., EUA, del 26 al 30 de septiembre del 2016. Resolución CD55.R10, Plan de acción para la reducción del riesgo de desastres 2016-2021: informe de progreso.

3. Cruz-Vega F, Loría-Castellanos J, Bautista Reyes AL, Montes de Oca García E. Aplicación de la capacitación en evacuación de unidades hospitalarias con énfasis en áreas críticas en el Instituto Mexicano del Seguro Social. Arch Med Urg Mex. 2016;8(1-2):7-13.

4. Cruz-Vega F, Loria-Castellanos J, et al. Evacuación de hospitales con énfasis en áreas críticas. Mèxico: Editorial Berit; 2018.

5. Cruz-Vega F, Elizondo-Argueta S, Sánchez-Echeverría JC, Loría-Castellanos J. Hospitales del Instituto Mexicano del Seguro Social durante los sismos de septiembre de 2017. Análisis desde la perspectiva del Programa Hospital Seguro. Gac Méd Méx. 2018;154(5):575-81.

6. Melgarejo-Urendez A, Bernat Adell MD, Lorente García P. Análisis de eventos adversos asociados al traslado intrahospitalario del paciente crítico. Listado de verificación. Enferm Intensiva. 2014;25(2):58-64.

7. Organización Panamericana de la Salud. Guía hospitalaria para la gestión de riesgo de desastres, convenio 344 de 2016, Bogotá, 12 de abril de 2017.

8. Organización Mundial de la Salud. Organización Panamericana de la Salud. Índice de seguridad hospitalaria. Guía para evaluadores. Segunda edición. Washington D.C.: Organización Mundial de la Salud, Organización Panamericana de la Salud.

9. World Health Organization. Safe hospitals in emergencies and disasters. Structural, non-structural and functional indicators save lives make hospitals safe in emergencies. World Health Organization; 2010.

10. Dirección de Prestaciones Médicas. Instituto Mexicano del Seguro Social. Plan institucional frente a emergencias y desastres: marco de actuación general. Cuarta ed. México: Instituto Mexicano del Seguro Social; 2018.

11. Loría-Castellanos J, Cruz-Vega F, Bautista Reyes AL, Saavedra-Gómez JL, Estevez-Abascal S, Montes de Oca García E. Características de incendios-conato de incendio en unidades hospitalarias del Instituto Mexicano del Seguro Social, 2012-2015. Perspectivas en urgencias. 2016;1:131-40.

12. Wong DF, Spencer C, Boyd L, Burkle FM, Archer F. Disaster metrics: A comprehensive framework for disaster evaluation typologies. Prehosp Disaster Med. 2017:32(5):501-14. Erratum en: Prehosp Disaster Med. 2017;32(4):469.

13. Curso de actualización para evaluadores vigentes [Internet]. México: Programa Hospital Seguro [consultado el 10 de enero del 2019]. Disponible en: https://hospitalseguro.org/e-learning/course/index.php

14. Bautista Reyes AL. ¿Programa Interno de Protección Civil o Plan Hospitalario para Emergencias y Desastres? Arch Med Urg Mex. 2015;7(1):1-3. 